\section{Slipping past P glycoprotein}

\section{By Kai-Jye Lou, Staff Writer}

NIH researchers have shown that sphingosine 1-phosphate receptor agonists such as Novartis AG's Gilenya fingolimod could transiently reduce P glycoprotein-mediated drug efflux and enhance drug delivery to the brain. ${ }^{1}$ The group at NIH's National Institute of Environmental Health Sciences thinks the transient mode of action of these agonists could avoid the adverse effects seen with direct $\mathrm{P}$ glycoprotein inhibitors.

The researchers now are elucidating the underlying mechanism and evaluating the approach for improving drug delivery to brain tumors.

$\mathrm{P}$ glycoprotein (MDR1; ABCB1; P-gp; CD243) is an active efflux transporter protein expressed by endothelial cells that line the blood brain barrier (BBB). The transporter exerts its neuroprotective effect by pumping out a range of potentially harmful molecules that enter the CNS from the blood. However, the transporter also complicates drug delivery to the CNS because it expels many therapeutics such as paclitaxel.

In cases in which a drug is a known substrate of P-gp, a straightforward strategy to enhance brain delivery is directly inhibiting $\mathrm{P}$-gp. The problem with this approach is that direct $\mathrm{P}$-gp inhibitors have caused unacceptable systemic toxicity in clinical trials, and nearly all such compounds have been discontinued.

Hanmi Pharmaceutical Co. Ltd. and partner Kinex Pharmaceuticals LLC are the only companies left with a disclosed oral P-gp inhibitor, HM30181A, but they are steering clear of the brain. The gastrointestinalselective P-gp inhibitor is in Phase II trials to treat gastric cancer in combination with an oral formulation of paclitaxel and is in Phase I testing to treat advanced solid malignancies in combination with oral irinotecan.

According to David Miller, head of the Intracellular Regulation Group and chief of the Laboratory of Toxicology \& Pharmacology at the National Institute of Environmental Health Sciences (NIEHS), the safety issues of direct P-gp inhibitors stem from the compounds being too potent and inhibiting P-gp's function for too long.

He proposed an alternative strategy to improve CNS drug delivery: to rapidly-but transiently-reduce P-gp efflux activity.

Miller and colleagues at NIEHS have been trying to elucidate the mechanisms that modulate the expression and activity of P-gp in cells along the $\mathrm{BBB}$ in hopes of finding new ways to target the transporter. For example, the group published a series of studies starting in 2004 detailing a tumor necrosis factor- $\alpha$ (TNF- $\alpha$ )-activated signaling pathway that reduces P-gp activity. ${ }^{2-4}$
Meanwhile, other academic groups have shown that TNF- $\alpha$ signaling in human endothelial cells stimulates the production of sphingosine 1-phosphate by activating sphingosine kinase 1 (SPHK1). ${ }^{5,6}$

Putting the puzzle pieces together, the NIEHS group sought to determine whether sphingosine 1-phosphate signaling itself could reduce P-gp efflux activity.

In an in vitro assay using isolated rodent brain capillaries, $1 \mu \mathrm{M}$ of sphingosine 1-phosphate caused complete loss of P-gp activity within 30 minutes. Subsequent removal of the lipid metabolite led to restoration of P-gp function within the same time frame. The researchers also showed that sphingosine 1-phosphate acts further downstream than previously identified members of the TNF- $\alpha$-activated pathway.

"We found that sphingosine 1-phosphate receptor agonists rapidly and reversibly modulate the activity of P-gp to reduce its transport of drugs out of the blood brain barrier," said Miller. "In our in vitro assay, we see a reduction in efflux activity within 5-10 minutes, with the full effect being achieved within 30 minutes, and we see that the time course of recovery after removing the agonist is also on the order of 30 minutes."

According to Miller, going after targets that are closer to the point of P-gp modulation should have a lower risk of side effects than hitting more upstream targets.

In rats, sphingosine 1-phosphate receptor agonists such as Gilenya increased BBB penetration of paclitaxel and the opioid receptor agonist loperamide by up to fivefold compared with delivering the two drugs without an agonist.

Results were published in the Proceedings of the National Academy of Sciences.

Patrick Ronaldson, an assistant professor in the Department of Pharmacology at The University of Arizona College of Medicine, noted that the rapid and transient mode of action should enable much finer control over drug delivery to the brain than earlier P-gp inhibitors.

Novartis markets Gilenya to treat relapsing forms of multiple sclerosis (MS). The pharma was not involved in the study and declined to comment on the findings.

\section{Safety first}

It remains unclear whether Gilenya itself would be a good sphingosine 1-phosphate agonist to use to enhance the brain delivery of other drugs.

Pieter Gaillard, cofounder and CSO at to-BBB technologies B.V., noted that Gilenya has significant side effects.

"It is very interesting that this drug has such an effect on drug delivery to the CNS in addition to its known immunomodulatory effects," he told SciBX. "But the drug itself is already associated with cardiotoxicity and infection risk, and it isn't yet clear how widespread such effects would be."

Gaillard said the safety concerns would be magnified by the need for high plasma concentrations to improve drug delivery across the BBB.

Miller said he is aware of the safety signals associated with Gilenya and acknowledged that the plasma concentrations in patients with MS who take the drug orally are at least an order magnitude lower than what is needed to enhance drug delivery across the BBB.

"If we are to use fingolimod, we would probably want to deliver 
the drug via carotid infusion to get the high local concentrations needed to enhance drug delivery across the blood brain barrier," he said. "This also has the benefit of having a short-term effect as fingolimod will then be diluted into systemic circulation, so its effects on P-gp should quickly wear off after stopping the infusion."

Gaillard agreed that carotid infusion could be a viable approach to achieve the high local concentrations needed, but he said the approach is more invasive than i.v. delivery and thus could restrict its use.

to-BBB uses liposomes coated with glutathione-conjugated polyethylene glycol (PEG) to improve drug delivery across the BBB. The biotech's 2B3-101, a liposomal formulation of doxorubicin coated with glutathione-conjugated PEG, is in Phase I/IIa testing to treat brain cancer.

\section{Picking an indication}

Miller said the NIEHS group is starting to evaluate the Pieter Gaillard agonist strategy in a rodent brain tumor model.

Because Gilenya also has immunomodulatory effects, Gaillard said it will be important for the NIEHS researchers to evaluate their approach in oncology models that include the ability to measure effects on the immune system.

"Other disease areas to consider for this approach could be multiple sclerosis, Parkinson's disease, Alzheimer's disease and possibly epilepsy, as many antiepileptics are known to be difficult to deliver to the brain," Ronaldson told SciBX. "One other area would be in HIV where the infection has spread to the brain, as current antiretroviral drugs also have trouble getting into the brain."

Ronaldson said dose-response studies are going to be important because the NIEHS group will need to determine what type of dosing adjustments are needed for existing drugs when they are co-delivered with a sphingosine 1-phosphate receptor agonist. Moreover, he said that it will be important to determine what other efflux and influx transporter proteins might be affected by sphingosine 1-phosphate receptor agonists.

In addition to the drug delivery studies, Miller said the group is looking downstream of the sphingosine 1-phosphate receptors to identify targets that are even closer to the observed down-modulation of P-gp-mediated drug efflux.

"This may also lead us to identify another transporter that we can modulate to enhance CNS drug delivery," he added.

The work in the paper is unpatented.

Lou, K.-J. SciBX 5(39); doi:10.1038/scibx.2012.1019

Published online Oct. 4, 2012

\section{REFERENCES}

1. Cannon, R.E. et al. Proc. Natl. Acad. Sci. USA; published online Sept. 4, 2012; doi:10.1073/pnas.1203534109

Contact: Ronald E. Cannon, National Institute of Environmental Health Sciences, National Institutes of Health, Research Triangle Park, N.C. e-mail: cannon1@niehs.nih.gov

2. Hartz, A.M.S. et al. Mol. Pharmacol. 66, 387-394 (2004)

3. Hartz, A.M.S. et al. Mol. Pharmacol. 69, 462-470 (2006)

4. Rigor, R.R. et al. J. Cereb. Blood Flow Metab. 30, 1373-1383 (2010)

5. Xia, P. et al. J. Biol. Chem. 274, 34499-34505 (1999)

6. De Palma, C. et al. Arterioscler. Thromb. Vasc. Biol. 26, 99-105 (2006)

\section{COMPANIES AND INSTITUTIONS MENTIONED}

Hanmi Pharmaceutical Co. Ltd. (KOSDAQ:128940), Seoul, South Korea

Kinex Pharmaceuticals LLC, Buffalo, N.Y. National Institute of Environmental Health Sciences, Research Triangle Park, N.C.

National Institutes of Health, Bethesda, Md.

Novartis AG (NYSE:NVS; SIX:NOVN), Basel, Switzerland

to-BBB technologies B.V., Leiden, the Netherlands

The University of Arizona College of Medicine, Tucson, Ariz. 